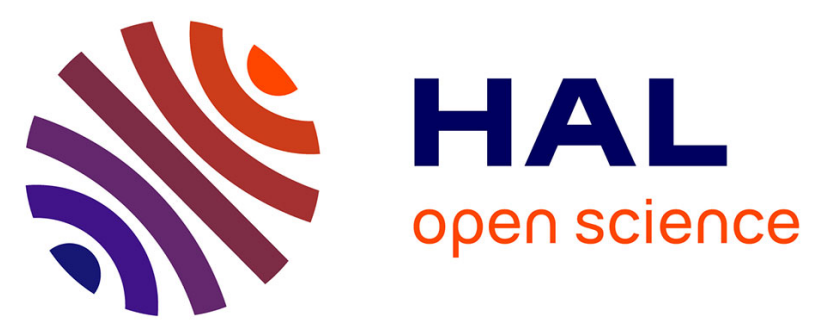

\title{
Buckling of Viscous Filaments of a Fluid under Compression Stresses
}

Marie Le Merrer, David Quéré, Christophe Clanet

\section{To cite this version:}

Marie Le Merrer, David Quéré, Christophe Clanet. Buckling of Viscous Filaments of a Fluid under Compression Stresses. Physical Review Letters, 2012, 109 (6), pp.064502. 10.1103/physrevlett.109.064502 . hal-00996501

\section{HAL Id: hal-00996501 \\ https://hal-polytechnique.archives-ouvertes.fr/hal-00996501}

Submitted on 5 Jun 2014

HAL is a multi-disciplinary open access archive for the deposit and dissemination of scientific research documents, whether they are published or not. The documents may come from teaching and research institutions in France or abroad, or from public or private research centers.
L'archive ouverte pluridisciplinaire HAL, est destinée au dépôt et à la diffusion de documents scientifiques de niveau recherche, publiés ou non, émanant des établissements d'enseignement et de recherche français ou étrangers, des laboratoires publics ou privés. 


\title{
Buckling of Viscous Filaments of a Fluid under Compression Stresses
}

\author{
Marie Le Merrer, ${ }^{1,2,3}$ David Quéré, ${ }^{1,2}$ and Christophe Clanet ${ }^{1,2}$ \\ ${ }^{1}$ PMMH, UMR 7636 CNRS-ESPCI-Université Paris 6-Université Paris 7, 75005 Paris, France \\ ${ }^{2}$ LadHyx, UMR 7646 CNRS-École Polytechnique, 91120 Palaiseau, France \\ ${ }^{3}$ INSP, UMR 7588 CNRS-Université Paris 6, 75005 Paris, France
}

(Received 8 February 2012; revised manuscript received 16 May 2012; published 10 August 2012)

\begin{abstract}
We study the compression of viscous filaments at constant velocity. If slender enough, the filament bends, a viscous analogue of Euler elastic buckling. We measure the characteristic time of this viscous buckling and discuss the link with the elastic critical compression. We show that the analogy only holds in the limit of large capillary numbers. Otherwise capillarity has a stabilizing effect, which suppresses buckling.
\end{abstract}

DOI: 10.1103/PhysRevLett.109.064502

PACS numbers: 47.20.Gv, 46.32.+x, 47.55.N-

Since Euler's elastica [1], elastic buckling has been widely studied and has become a classical field in solid mechanics [2]; beyond a critical compression, a solid rod bends, as the energy of flexure becomes smaller than the cost of compression. Besides the theoretical similarity underlined by Stokes and Rayleigh [3,4], there is evidence that liquid rods can behave like solid ones if viscous enough [5]. For example, such filaments in the field of gravity adopt the shape of solid catenaries [6-8], while they coil as ropes when hitting a substrate $[9,10]$. Similarly, viscous jets or sheets can sometimes fold [11-21], and this effect is suspected to be the cause of irregularities in large glass plates made from molten glass [22] and responsible for folded geological structures [15,23]. Here, we wonder whether liquid rods also buckle when compressed and discuss the criteria for observing this phenomenon.

By stretching a drop of viscosity $\eta$ and surface tension $\gamma$, we first create a vertical filament of length $L$ and diameter $D$. The high viscosity prevents the thread from undergoing Rayleigh instability and breaking into several droplets [24]. Then, the lower part of the stretching device is pushed back towards the upper part at a constant velocity $U$. The experiment is recorded from the side, and we display in Fig. 1 two image sequences showing the compression of vertical threads of initial length $L=8 \mathrm{~mm}$, diameter $D=0.6 \mathrm{~mm}$, and made of silicon oil of viscosity $\eta=500 \mathrm{~Pa} \cdot \mathrm{s}$ (i.e., $5 \times 10^{5}$ times the viscosity of water). The behavior of the filament is very different, according to the compression velocity $U$. For $U=200 \mu \mathrm{m} / \mathrm{s}$ [Fig. 1(a)], the liquid cylinder simply becomes shorter and thicker, a consequence of its liquid nature. The same filament compressed at $U=1 \mathrm{~mm} / \mathrm{s}$ [Fig. 1(b)] starts to bend after one second: the liquid thread buckles as a solid rod. The Reynolds number $\rho U L / \eta$ (where $\rho$ is the liquid density) is of order $10^{-5}$ in this experiment. Because of the existence of free surfaces, the Stokes flow loses time reversibility [25]: compression is not simply the reverse of stretching but generates buckling. However, contrasting with the example of honey coiling, gravity plays no role here, as deduced from the up-down symmetry of the filament in Fig. 1. This low gravity regime is achieved when the Laplace pressure $2 \gamma / D$ exceeds the hydrostatic pressure $\rho g L / 2$. This condition is satisfied for $L D<4 a^{2}$, where $a=\sqrt{\gamma / \rho g}$ is the capillary length $(1.5 \mathrm{~mm}$ for silicon oil), i.e., for short or thin filaments, like in all the experiments reported here.

We first focus on what happens at high compression velocity [Fig. 1(b)]. When the filament buckles, we measure the time evolution of the off-axis deflection $\delta$ of the centerline of the filament, defined in Figs. 1 and 4. Figure 2 shows the results for filaments of different lengths. Two regimes are observed. At short time, the thread remains axisymmetric $(\delta=0)$, and it thickens; after some delay $(t>\tau), \delta$ increases with $t$. The deflection then varies as the

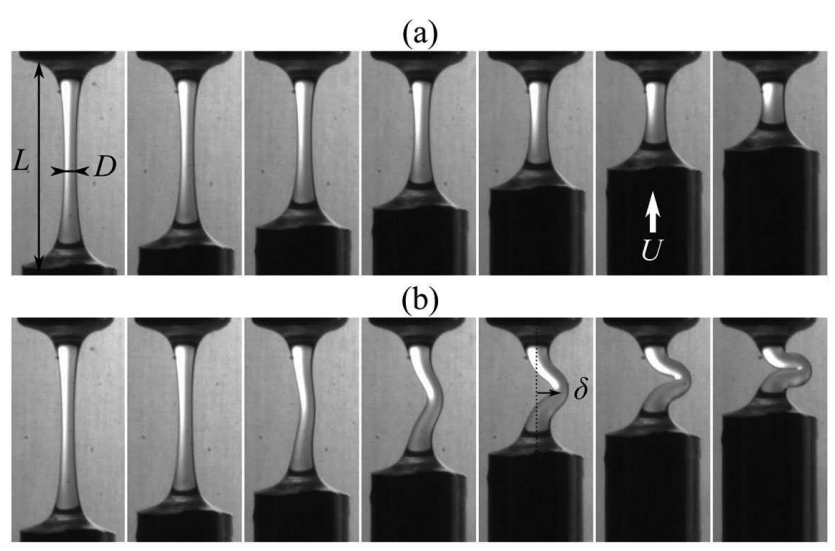

FIG. 1. Image sequences of a viscous filament $(\eta=500$ Pa.s, $\gamma=20 \mathrm{mN} / \mathrm{m}$ ) compressed at a constant velocity $U$. The initial length of the thread is $L=8 \mathrm{~mm}$, and its initial diameter $D=0.6 \mathrm{~mm}$. Since the diameter is not homogeneous along the filament, we chose to denote $D$ the minimal diameter, measured at the middle of the thread. (a) $U=200 \mu \mathrm{m} / \mathrm{s}$, which corresponds to $\mathrm{Ca}=\eta U / \gamma \approx 5$; the interval $\Delta t$ between two images is $4 \mathrm{~s}$; (b) $U=1 \mathrm{~mm} / \mathrm{s}$ and $\mathrm{Ca} \approx 25 ; \Delta t=0.8 \mathrm{~s}$. The filament bends after some delay, and we measure its off-axis deflection $\delta$ as a function of time. 


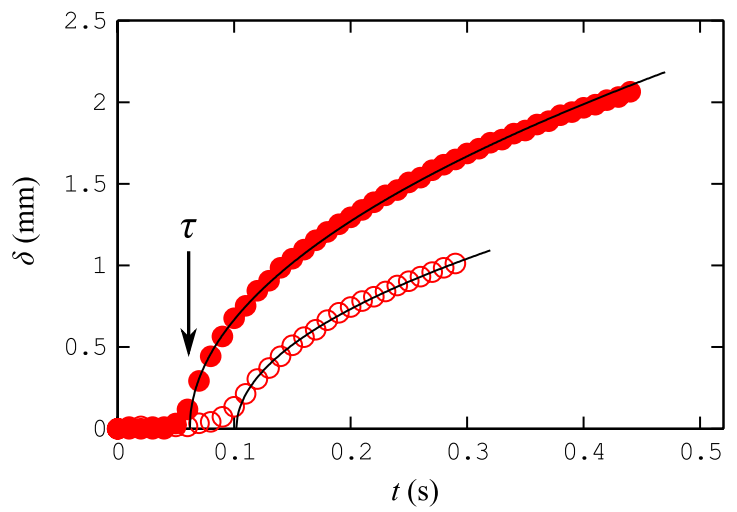

FIG. 2 (color online). Deflection $\delta$ as a function of time $t$. The data have been obtained for $\eta=10^{3} \mathrm{~Pa} \cdot \mathrm{s}, D=0.4 \mathrm{~mm}$, $U=5 \mathrm{~mm} / \mathrm{s}$, and two initial lengths: $L=11 \mathrm{~mm}$ (solid dots) and $L=5 \mathrm{~mm}$ (open dots). The solid lines correspond to $\delta \propto \sqrt{t-\tau}$.

square root of time (solid lines). This is a geometrical consequence of the conservation of filament length after $\tau$, which imposes $\delta^{2} \sim L U(t-\tau)$ for $t \ll L / U$.

The time $\tau$ [extracted by extrapolating the square root behavior of $\delta(t)$ down to $\delta=0$ ] can be seen as a characteristic time of viscous buckling. Figure 2 indicates that $\tau$ decreases with $L$, and Fig. 3 shows that it increases with the initial diameter $D$ of the thread: slender bodies tend to buckle earlier. Figure 3 also shows that $\tau$ decreases with the compression velocity $U$.

Before trying to understand viscous buckling, let us first remind ourselves of the case of an elastic rod of Young modulus $E$, compressed by a distance $x$, which slightly deviates from its axis [Fig. 4(a)]. The compressive force $F \sim E D^{2} x / L$ tends to increase the deflection with a torque $F \delta$. When deflected by a quantity $\delta$, the rod is bent with a curvature $\kappa \sim \delta / L^{2}$ (for $\delta \ll L$ ). The bending stiffness scales as $E D^{4}$ so that the torque resisting bending is $E D^{4} \delta / L^{2}$. The two torques can be compared, and buckling

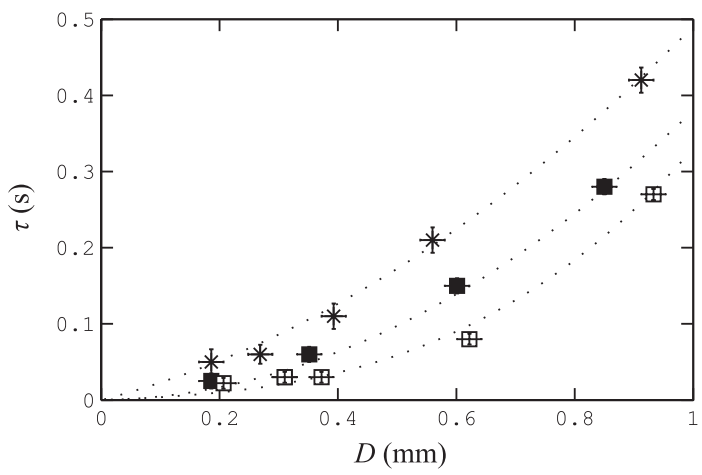

FIG. 3. Buckling time $\tau$ as a function of the diameter $D$ for $U=3 \mathrm{~mm} / \mathrm{s}(\times), \quad U=5 \mathrm{~mm} / \mathrm{s}$ (solid squares), and $U=7 \mathrm{~mm} / \mathrm{s}$ (open squares). The filament viscosity is $\eta=10^{3} \mathrm{~Pa} \cdot \mathrm{s}$, and its length $L=11 \mathrm{~mm}$. The dotted lines are guides for the eye. will be favored if $x>D^{2} / L$, that is, if the compressive force $F$ is higher than Euler's threshold $F_{c} \sim E D^{4} / L^{2}[1,2]$.

For a filament of viscosity $\eta$ compressed at a velocity $U$, the compressive viscous force is $(\eta U / L) D^{2}$; here, the length scale associated with the velocity gradient is $L$ because the flow is extensional due to the presence of free surfaces. The resulting torque is $(\eta U / L) D^{2} \delta$. We must also evaluate the nature of the torque opposing bending. For a filament bent by a curvature $\kappa$, the outer and inner parts are longer and shorter by a length $D L \kappa$ [Fig. 4(b)]. If the curvature changes, the rate of stretching (or compression) is $D \dot{\kappa}$, which implies a viscous stress $\eta D \dot{\kappa}$, hence, a viscous torque $\mathcal{M} \sim \eta D^{4} \dot{\kappa}$. This expression is similar to the elastic torque $E D^{4} \kappa$ resisting bending in a solid. This is a consequence of the similarity of viscous liquid and elastic solid constitutive equations [3,4]. Therefore, an analogy can be made between viscous and elastic effects in which $\eta \dot{\kappa}$ corresponds to $E \kappa$. In our situation, we have $\kappa \sim \delta / L^{2}$, and the torque balance can be written

$$
\eta D^{2} \frac{U}{L} \delta \sim \eta D^{4} \frac{\dot{\delta}}{L^{2}}
$$

This equation defines a characteristic time

$$
\tau_{0}=\frac{D^{2}}{L U}
$$

We plot in Fig. 5 the experimental buckling time $\tau$ as a function of $\tau_{0}$. The whole collection of data collapses on a single line of slope 20: $\tau_{0}$ is indeed the characteristic time for buckling. Since buckling can only be observed if $\tau$ is shorter than the experiment duration $L / U$, this leads to a first criterion for buckling: $D / L<1 / \sqrt{20} \approx 0.2$; only slender enough filaments are likely to buckle. It appears that the elastic buckling threshold $x \gtrsim D^{2} / L$ also holds for viscous filaments (apart from the numerical factor); in the latter case, we have $x=U t$, which yields, once introduced (a)

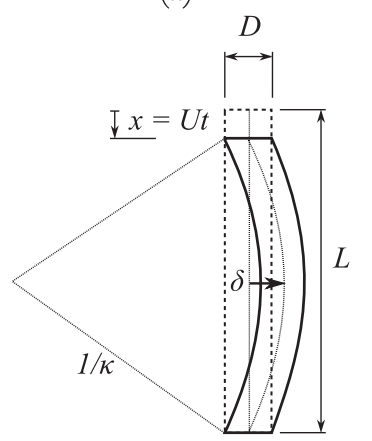

(b)

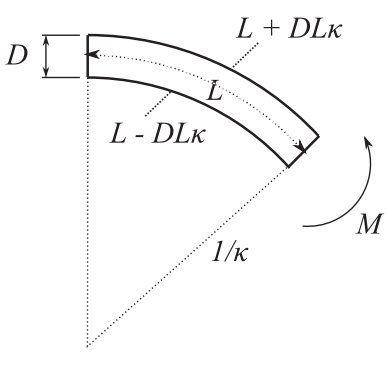

FIG. 4. (a) Under compression, a slender body (of length $L$ and diameter $D$ ) can bend and deviate from its axis. The deflection $\delta$ induces a curvature $\kappa \sim \delta / L^{2}$. (b) Sketch of a portion of filament (of length $L$ and diameter $D$ ) bent with a curvature $\kappa$. 


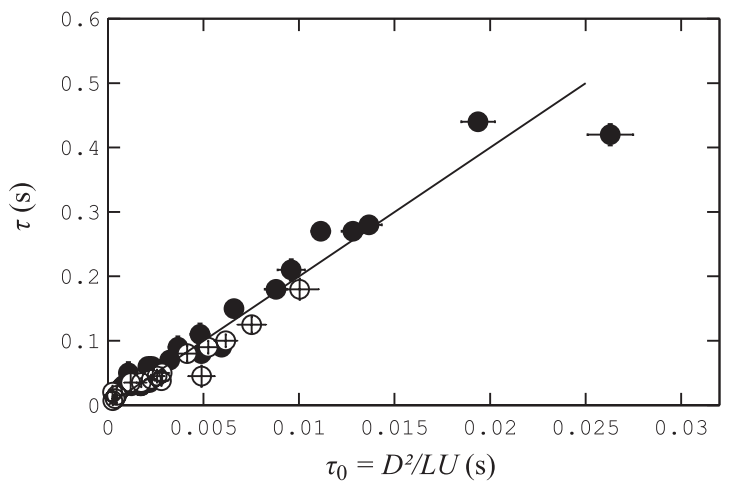

FIG. 5. Experimental time $\tau$ as a function of $\tau_{0}$. The open dots correspond to $L=5 \mathrm{~mm}$, the solid dots to $L=11 \mathrm{~mm}$. $D$ varies between 0.1 and $1 \mathrm{~mm}$ and $U$ between 3 and $7 \mathrm{~mm} / \mathrm{s}$. The silicon oil viscosity is $\eta=10^{3} \mathrm{~Pa} \cdot \mathrm{s}$. The straight line shows $\tau=20 \tau_{0}$.

in the elastic criterion, $t>\tau_{0}$. One might actually wonder whether the buckling phenomenon observed in our experiments could result from the elasticity of the polymer chains. However, the compression rate $U / L \sim 0.02-1 \mathrm{~s}^{-1}$ is always smaller than the critical shear rate $T^{-1} \sim$ $3-50 \mathrm{~s}^{-1}$ for shear thinning of the silicon oils, which confirms the dominant role of viscosity, as also observed with a purely viscous liquid such as honey (see Supplemental Material [26]).

Equation (1) predicts that the filament is intrinsically unstable [27], with an exponential growth for $\delta$. However, the fully developed exponential regime cannot be seen in the experiments because stretching soon dominates bending: Eq. (1) is valid only if the stretching rate $\delta \dot{\delta} / L^{2}$ is smaller than $D \dot{\kappa} \sim D \dot{\delta} / L^{2}$, i.e., for $\delta<D$. If $\delta_{0}$ denotes the initial deflection, $\delta=D$ is reached after a time $\tau \sim \tau_{0} \ln \left(D / \delta_{0}\right)$. Taking the scale of thermal fluctuations for $\delta_{0}(\sim \sqrt{k T / \gamma} \sim 1 \mathrm{~nm})$ and $D \sim 1 \mathrm{~mm}$, we find $\tau \approx 14 \tau_{0}$. Later on, for $\delta>D, \delta$ is set by the finite length of the filament and varies as the square root of time, as seen earlier.

This elasticity-viscosity analogy holds in the limit of dominant viscous effects. Surface tension, which was neglected in the derivation of Eq. (1), has a stabilizing effect on buckling [28,29]: an axisymmetric shape has less free surface than a buckled shape. This effect can be expressed as a stretching capillary force $\gamma D$ driving the liquid towards the ends of the filament. Surface tension will be negligible when $\gamma D$ is smaller than the compressive viscous force $(\eta U / L) D^{2}$, i.e., for

$$
\frac{D}{L} \frac{\eta U}{\gamma}>1
$$

Logically, this new criterion depends on the capillary number $\mathrm{Ca}=\eta U / \gamma$. It implies that buckling should disappear at small velocities, as indeed observed in Fig. 1(a). (a)

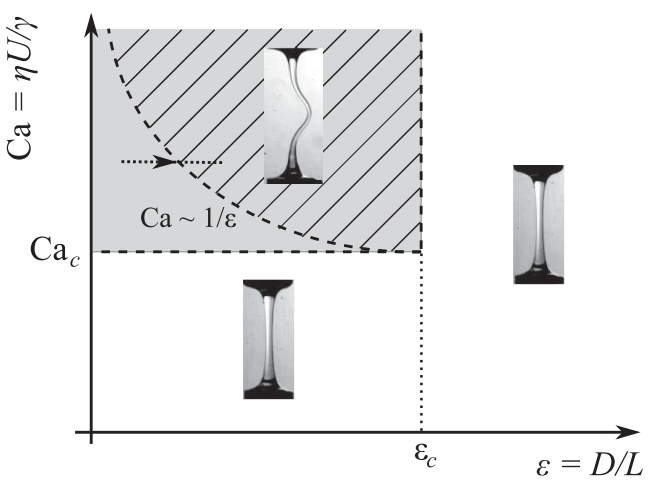

(b)

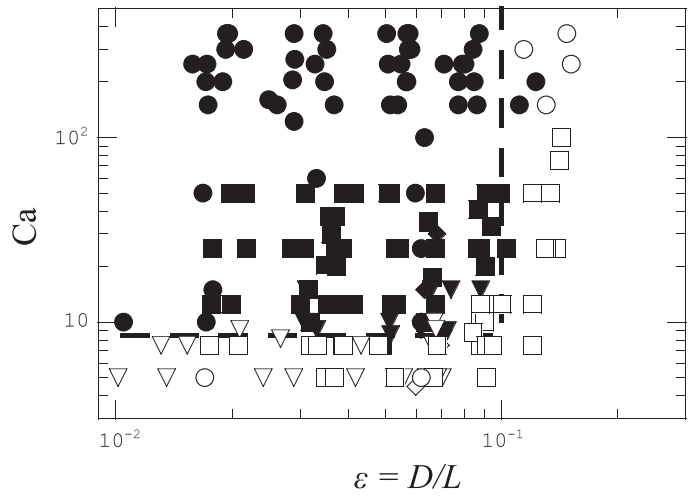

FIG. 6. (a) Phase diagram for viscous buckling. The hatched area is the region where buckling is expected, following the two criteria $\epsilon<\epsilon_{c}$ and $\mathrm{Ca} \epsilon>1$. In an experiment, $\epsilon$ increases at fixed $\mathrm{Ca}$, following the dotted line. As this line enters the hatched region, buckling will occur. Therefore, the observable buckling region is the gray rectangle $\left(\epsilon<\epsilon_{c}, \mathrm{Ca}>1 / \epsilon_{c}\right)$. (b) Experimental phase diagram: each point corresponds to an experiment, and we report if there is buckling (solid symbols) or not (empty symbols). Our criterion for buckling is $\delta>D$ at the end of the experiment $(t \sim L / U)$. Symbols correspond to different oil viscosities: $(\nabla) \eta=10^{2} \mathrm{~Pa} \cdot \mathrm{s},(\diamond) \eta=3 \times 10^{2} \mathrm{~Pa} \cdot \mathrm{s}$, ( $\square) \eta=5 \times 10^{2} \mathrm{~Pa} \cdot \mathrm{s}$, (○) $\eta=10^{3} \mathrm{~Pa} \cdot \mathrm{s}$. Experiments done with viscous honey give similar results (see Supplemental Material [26]).

Therefore two conditions have to be fulfilled to observe buckling: (i) the filament must be slender enough [Eq. (2) and below, $\epsilon \equiv D / L<0.2$ ]; (ii) surface tension has to be negligible [Eq. (3)]. These two criteria can be summarized in the $(\epsilon, \mathrm{Ca})$ phase diagram in Fig. $6(\mathrm{a})$, in which the hatched area is the region of buckling expected from these criteria. However, as long as there is no deflection, the ratio $\epsilon$ in an experiment increases at fixed capillary number, following the dotted line in the diagram. If $\mathrm{Ca}$ is high enough [gray region in Fig. 6(a)], one will eventually enter the region for buckling. Consequently, buckling should be observed for slender enough filaments $\left(\epsilon<\epsilon_{c} \sim 10^{-1}\right)$ and at quick enough compressions, that is, $\mathrm{Ca} \gtrsim \mathrm{Ca}_{c} \sim$ $\epsilon_{c}^{-1} \sim 10$, in excellent agreement with the experimental 
phase diagram in Fig. 6(b). In the $(\epsilon, \mathrm{Ca})$ graph, it is clearly observed that the buckling region is the top-left part of the diagram, with $\epsilon_{c} \approx 10^{-1}$ and $\mathrm{Ca}_{c} \approx 10$. For the experiments of Fig. $1(\eta=500 \mathrm{~Pa} \cdot \mathrm{s}$ and $\gamma \approx 20 \mathrm{mN} / \mathrm{m})$, this corresponds to a minimum buckling velocity $U_{c}=0.4 \mathrm{~mm} / \mathrm{s}$. Note, finally, that this picture holds if inertia is negligible. Taking $U=U_{c}=10 \gamma / \eta$ and a Reynolds number smaller than 1 , this is expected for $\eta \gtrsim 1 \mathrm{~Pa} \cdot \mathrm{s}$, a condition always fulfilled in this study.

As a conclusion, experiments and models confirm the strength of the analogy between elastic and viscous buckling: this phenomenon is observed when compressing quick enough slender viscous filaments. This simple experiment also provides some insight on the physics of other viscous buckling phenomena, such as the coiling of a viscous jet, a more complex situation since gravity breaks the up-down symmetry of the jet; for thin jets (when surface tension is not negligible), the minimum jet height for buckling is proportional to its diameter, as expected from criterion (i), except for low injection velocities [criterion (ii)], for which the jet height (and thus the importance of gravity) has to be increased [11]. It would be interesting in future work to consider the buckling of filaments when $L D \geqslant a^{2}$ and compare it to the coiling of thick jets, for which viscous-dominated or gravitydominated buckling criteria are expected [10].

We would like to thank Arezki Boudaoud, Neil Ribe, Anke Lindner, and Benoît Roman for stimulating discussions.

[1] L. Euler, Methodus Inveniendi Lineas Curvas Maximi Minimive Proprietate Gaudentes, Additamentum I (De Curvis Elasticis), Leonardi Euleri Opera Omnia Vol. 24 (Birkhäuser, Basel, 1744), p. 245.

[2] S.P. Timoshenko and J.M. Gere, Theory of Elastic Stability (McGraw-Hill, New York, 1963).

[3] G. G. Stokes, Trans. Cambridge Philos. Soc. 8, 287 (1845).

[4] J. W. S. Rayleigh, The Theory of Sound (Dover, New York, 1945), Vol. 2, p. 313.

[5] G. I. Taylor, in Proceedings of the Twelfth International Congress of Applied Mechanics, Stanford, 1968 (SpringerVerlag, Berlin, 1969), p. 382.
[6] J. Teichman and L. Mahadevan, J. Fluid Mech. 478, 71 (2003).

[7] J. P. Koulakis, C. D. Mitescu, F. Brochard-Wyart, P.-G. de Gennes, and E. Guyon, J. Fluid Mech. 609, 87 (2008).

[8] M. Le Merrer, J. Seiwert, D. Quéré, and C. Clanet, Europhys. Lett. 84, 56004 (2008).

[9] L. Mahadevan, W.S. Ryu, and A.D. T. Samuel, Nature (London) 392, 140 (1998); 403, 502 (2000).

[10] N. M. Ribe, M. Habibi, and D. Bonn, Annu. Rev. Fluid Mech. 44, 249 (2012).

[11] J. O. Cruickshank and B. R. Munson, J. Fluid Mech. 113, 221 (1981).

[12] M. Skorobogatiy and L. Mahadevan, Europhys. Lett. 52, 532 (2000)

[13] N. M. Ribe, J. Fluid Mech. 433, 135 (2001).

[14] N. M. Ribe, Phys. Rev. E 68, 036305 (2003).

[15] M. A. Biot, Phys. Fluids 7, 855 (1964).

[16] S. M. Suleiman and B. R. Munson, Phys. Fluids 24, 1 (1981).

[17] T. B. Benjamin and T. Mullin, J. Fluid Mech. 195, 523 (1988).

[18] G. Debrégeas, P.-G. de Gennes, and F. Brochard-Wyart, Science 279, 1704 (1998).

[19] R. da Silveira, S. Chaieb, and L. Mahadevan, Science 287, 1468 (2000).

[20] A. Boudaoud and S. Chaïeb, Phys. Rev. E 64, 050601 (2001).

[21] T. Cubaud and T. G. Mason, Phys. Rev. Lett. 96, 114501 (2006).

[22] L. A. B. Pilkington, Proc. R. Soc. A 314, 1 (1969).

[23] A. M. Johnson and R.C. Fletcher, Folding of Viscous Layers: Mechanical Analysis and Interpretation of Structures in Deformed Rock (Columbia University, New York, 1994).

[24] J. W. S. Rayleigh, Philos. Mag. 34, 145 (1892).

[25] R. Trouilloud, T. S. Yu, A. E. Hosoi, and E. Lauga, Phys. Rev. Lett. 101, 048102 (2008).

[26] See Supplemental Material at http://link.aps.org/ supplemental/10.1103/PhysRevLett.109.064502 for a description of buckling experiments performed with viscous honey.

[27] J.D. Buckmaster, A. Nachman, and L. Ting, J. Fluid Mech. 69, 1 (1975).

[28] J.D. Buckmaster and A. Nachman, Q. J. Mech. Appl. Math. 31, 157 (1978).

[29] T. Cubaud, B. M. Jose, and S. Darvishi, Phys. Fluids 23, 042002 (2011). 